\title{
Airway dendritic cell phenotypes in inflammatory diseases of the human lung
}

\author{
M. Lommatzsch*,\#, K. Bratke*, , A. Bier*, P. Julius*, M. Kuepper*, \\ W. Luttmann* and J.C. Virchow*
}

ABSTRACT: Airway dendritic cells (DCs) are key regulators of pulmonary immune responses. However, information is limited regarding the characteristics of airway DCs in human lung diseases.

Plasmacytoid DCs (pDCs) and myeloid DCs (mDCs) were analysed using four-colour flow cytometry in bronchoalveolar lavage fluid (BALF) from nonsmoking controls and patients with sarcoidosis, idiopathic pulmonary fibrosis (IPF) and pneumonia (in the presence or absence of immunosuppression).

Compared with controls, immunocompetent patients with pneumonia displayed strongly enhanced pDC counts in BALF. In contrast, pDC counts in BALF from immunocompromised patients with pneumonia were even lower than in controls. This discrepancy was not explained by a different chemotactic milieu in the airways; all patients with pneumonia were characterised by strongly increased concentrations of the PDC-attracting chemokine, CXC chemokine ligand 10, in BALF. Patients with IPF were characterised by normal percentages of DC subtypes. However, the mDCs of patients with IPF were not as mature (CD83-positive) as those of controls. Patients with sarcoidosis displayed a unique increase in CD1a-negative mDCs in the airways. In addition, there was altered expression of costimulatory molecules (increased CD80 and decreased CD86 expression) on mDCs in patients with sarcoidosis.

These data suggest that inflammatory diseases of the human lung are associated with a differential phenotype and recruitment of airway dendritic cells.

KEYWORDS: Bronchoalveolar lavage fluid, dendritic cells, idiopathic pulmonary fibrosis, pneumonia, sarcoidosis

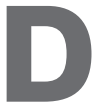
endritic cells (DCs) are professional antigen-presenting cells with a unique capacity to initiate and modulate specific immune responses [1]. In the lung, they form a highly sensitive sentinel network within and around the airways. By continuously reporting antigenic information from the airways to pulmonary lymph nodes, they are capable of upregulating or downregulating immune responses in the lung [2]. Human DCs are identified by abundant expression of major histocompatibility complex class II (human leukocyte antigen (HLA) DR) and the absence of lymphocyte, natural killer cell, monocyte and granulocyte lineage markers. They are currently classified into two developmentally and functionally distinct subsets: CD11c-positive myeloid DCs (mDCs), and CD123-positive plasmacytoid DCs (pDCs) [2]. CD1a-positive DCs have been identified as a subgroup of mDCs in human bronchoalveolar

For editorial comments see page 823 . lavage (BAL) fluid (BALF) [3]. These CD1apositive DCs appear to differ from CD1a-negative mDCs in their cytokine profile and their capacity to direct T-cell differentiation $[4,5]$.

Based on animal models, it has been postulated that airway DCs play a key role in diseases such as asthma [6]. However, information is limited regarding the role of DCs in the human lung. The currently available studies have described DCs in patients with asthma [3, 7-9], bronchiolitis [10] (COPD) [11]. In contrast, information concerning airway DCs in other inflammatory diseases of the human lung, and their relationship to healthy controls, is lacking. There are only reports describing the presence of DCs in lymph nodes of patients with sarcoidosis [12] and lung parenchyma of patients with interstitial pneumonias $[13,14]$. In patients with asthma, a comprehensive flow cytometric method has recently been established for the quantification and and chronic obstructive pulmonary disease
AFFILIATIONS

*Dept of Pneumology, University of Rostock, Rostock, Germany

\#Both authors contributed equally to this article.

CORRESPONDENCE

M. Lommatzsch

Abteilung für Pneumologie

Klinik und Poliklinik für Innere

Medizin

Universität Rostock

Ernst-Heydemann-Str. 6

18057 Rostock

Germany

Fax: 493814947392

E-mail: marek.lommatzsch@

med.uni-rostock.de

Received:

March 262007

Accepted after revision:

June 272007

SUPPORT STATEMENT

This study was supported by German

Research Foundation (Deutsche

Forschungsgemeinschaft; Bonn,

Germany) grant L0 1145/2-1.

STATEMENT OF INTEREST

None declared. 
characterisation of DCs in human BALF [3]. Using this method, the present study describes, for the first time, the phenotype of airway DCs in patients with sarcoidosis, idiopathic pulmonary fibrosis (IPF), pneumonia and healthy controls.

\section{METHODS}

\section{Participants}

The BALF of 106 consecutive patients who were referred to the Dept of Pneumology (University of Rostock, Rostock, Germany) for suspected sarcoidosis, interstitial lung disease or pneumonia was examined. Of these patients, 35 fulfilled the present criteria for sarcoidosis, IPF or pneumonia, and were included in the current study. Patients were diagnosed as having sarcoidosis if they displayed typical clinical and radiological signs (stage I or II) of sarcoidosis, no evidence in transbronchial lung biopsy specimens or BALF of tuberculosis or other pulmonary diseases, and an enhanced percentage $(>20)$ of lymphocytes in BALF, with a CD4:CD8 ratio of $>3$. Patients were diagnosed as having IPF based on the American Thoracic Society/European Respiratory Society consensus criteria [15], as follows: 1) exclusion of other causes of pulmonary fibrosis; 2) restriction and impaired gas exchange on pulmonary function testing; 3 ) bibasilar reticular abnormalities on high-resolution computed tomography, compatible with usual interstitial pneumonitis; and 4) transbronchial lung biopsy specimens and BALF showing no features to support an alternative diagnosis. Patients were diagnosed as having pneumonia based on the following criteria: 1) onset of dyspnoea and/or cough within the 14 days prior to bronchoscopy; 2) radiological and clinical signs suggesting pneumonia; and 3) enhanced serum levels of C-reactive protein $\left(>5 \mu \mathrm{g} \cdot \mathrm{mL}^{-1}\right)$. Immunosuppression was defined as the presence of chemotherapy-induced neutropenia $\left(<0.5 \times 10^{6}\right.$ neutrophils $\cdot \mathrm{mL}$ blood $\left.^{-1}\right)$ and/or treatment with immunosuppressive agents (corticosteroids, cyclosporin or mycophenolate mofetil). As controls, 12 healthy nonsmoking volunteers (inclusion criteria: no history of smoking, no history of respiratory diseases and no regular medication) were recruited. All participants gave their written informed consent. The study was approved by the local ethics committee of Rostock.

\section{Bronchoalveolar lavage}

BAL was performed using flexible bronchoscopes (Olympus, Hamburg, Germany). The bronchoscope was wedged into a subsegment of the right middle lobe (in controls and patients with sarcoidosis or IPF) or an affected lobe (in patients with pneumonia), and a total of $100 \mathrm{~mL}$ pre-warmed sterile saline was instilled. The BALF was recovered by gentle aspiration.

\section{Flow cytometry and ELISA}

BALF samples were filtered through two layers of sterile gauze into sterile plastic vials, and centrifuged for $10 \mathrm{~min}$ at $500 \times \mathrm{g}$ at $4{ }^{\circ} \mathrm{C}$. Supernatants were aliquoted for ELISA and the cells were resuspended in PBS ( $\mathrm{pH}$ 7.2). BALF differential cell counts were determined as previously described [3]. Freshly isolated BALF cells were analysed using four-colour flow cytometry as previously described [3], using the antibody panel detailed in table 1 . The specific gating of DCs in BALF is detailed for each of the diseases and the controls in figure 1. Surface molecules were quantified in histogram plots using appropriate isotype control antibodies to discriminate between specific and nonspecific antibody staining (data not shown). CC chemokine ligand (CCL) 20 and CXC chemokine ligand (CXCL) 10 concentrations were measured in BALF supernatants using ELISA, according to the instructions of the manufacturer (R\&D Systems, Minneapolis, MN, USA).

\section{Statistical analysis}

Most parameters were non-normally distributed. Therefore, correlation analysis was performed using Spearman's correlation coefficient, and comparison of BALF parameters between groups was performed using the Mann-Whitney U-test. A pvalue of $<0.05$ was regarded as significant. The percentage of CD1a-negative mDCs was calculated by subtracting the percentage of CD1a-positive DCs from the percentage of CD11c-positive DCs.

\section{RESULTS}

\section{Patient characteristics}

The number, age and sex of the participants, as well as the BALF characteristics, are detailed in table 2. There was a positive smoking history in one patient with sarcoidosis (former smoker), four patients with IPF (two former and two current smokers), two patients of the pneumonia subgroup with immunosuppression (former smokers) and two patients of the pneumonia subgroup without immunosuppression (current smokers). None of the patients with a positive

\section{TABLE 1 Antibodies used for four-colour flow cytometry}

\begin{tabular}{lccc} 
Antigen & Label & Clone & Company \\
\hline Lineage cocktail & & & \\
CD3 & FITC & SK7 & BD Biosciences \\
CD14 & FITC & M 9 P9 & BD Biosciences \\
CD16 & FITC & $3 G 8$ & BD Biosciences \\
CD19 & FITC & SJ25C1 & BD Biosciences \\
CD20 & FITC & L27 & BD Biosciences \\
CD56 & FITC & NCAM16.2 & BD Biosciences \\
Other antibodies & & & \\
CD11C & PE & S-HCL-3 & BD Biosciences \\
CD11c & APC & S-HCL-3 & BD Biosciences \\
CD123 & PE & $9 F 5$ & BD Biosciences \\
HLA-DR & PerCP & L243 & BD Biosciences \\
CD40 & APC & HB14 & Caltag/lnvitrogen \\
CD83 & APC & HB15e & Caltag/lnvitrogen \\
CD86 & APC & BU63 & Caltag/lnvitrogen \\
CD80 & APC & MEM-233 & Immunotools \\
CD1a & PE & NA1/34 & Dako \\
BDCA-1 & APC & AD5-8E7 & Miltenyi Biotec \\
BDCA-2 & APC & AC144 & Miltenyi Biotec \\
BDCA-3 & APC & AD5-14H12 & Miltenyi Biotec \\
BDCA-4 & APC & AD5-17F6 & Miltenyi Biotec \\
\hline
\end{tabular}

HLA: human leukocyte antigen; BDCA: blood dendritic cell antigen; FITC: fluorescein isothiocyanate; PE: phycoerythrin; APC: allophycocyanin; PerCP: peridinin chlorophyll protein. Company locations are as follows: BD Biosciences, Heidelberg, Germany; Caltag/Invitrogen, Karlsruhe, Germany; Immunotools, Friesoythe, Germany; Dako, Glostrup, Denmark; and Milteny Biotec, Bergisch Gladbach, Germany. 

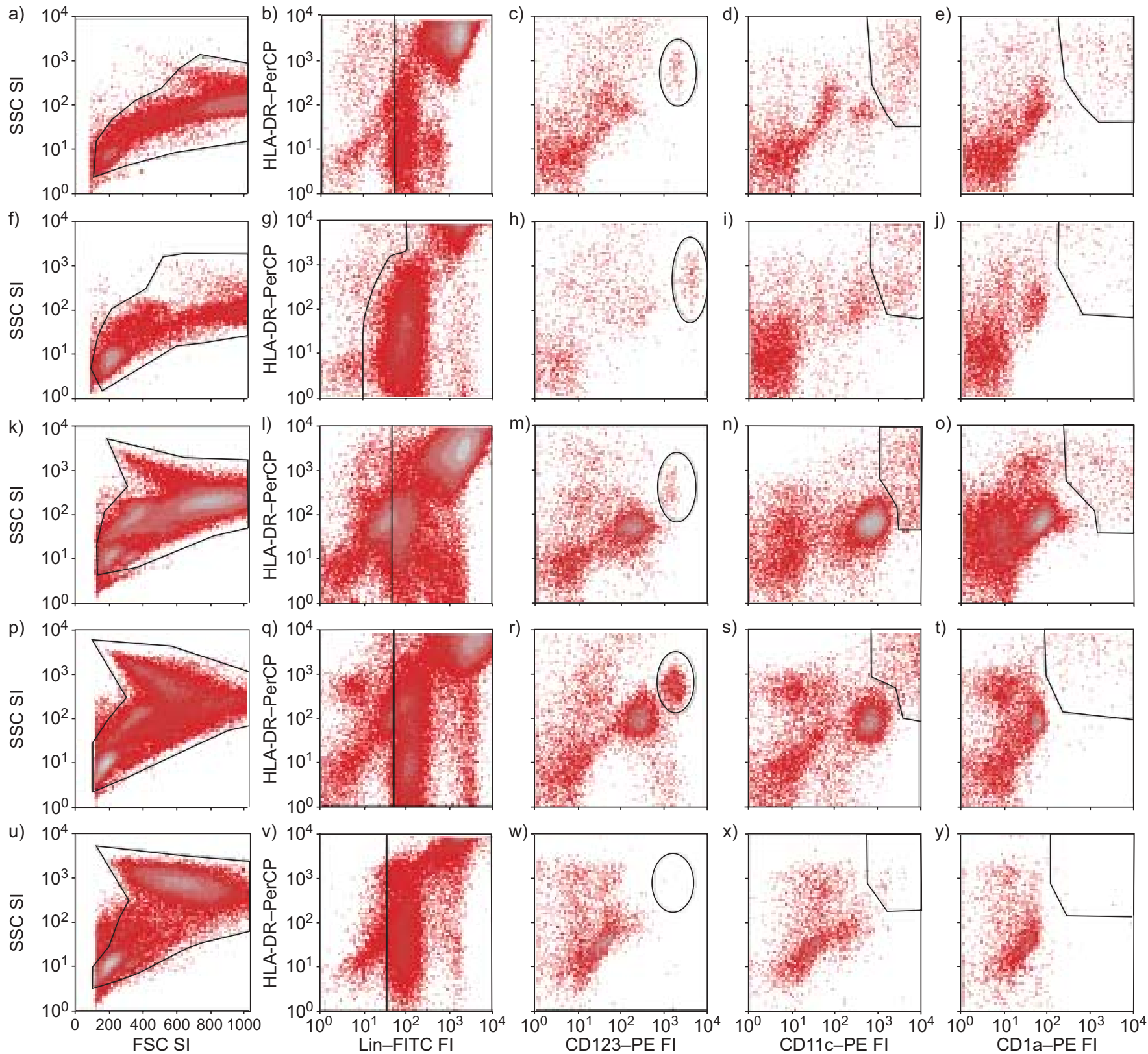

FIGURE 1. Gating (—_: selected areas) of dendritic cell (DC) subsets in bronchoalveolar lavage fluid (BALF) in: a-e) controls; f-j) sarcoidosis patients; $k-0)$ idiopathic pulmonary fibrosis; $\mathrm{p}-\mathrm{t}$ ) pneumonia without immunosuppression; and $\mathrm{u}-\mathrm{y}$ ) pneumonia during immunosuppression. Total BALF cells were identified in a forward scatter

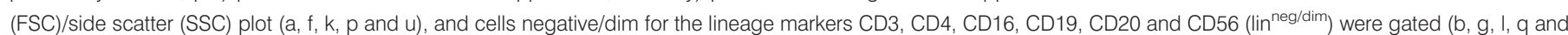
v). Among the lin ${ }^{\text {neg/dim }}$ cells, plasmacytoid DCs were identified by CD123 and human leukocyte antigen (HLA)-DR coexpression (c, h, m, r and w), myeloid DCs by CD11c and HLA-DR coexpression ( $d, i, n, s$ and $x$ ), and CD1a-positive DCs by CD1a and HLA-DR coexpression (e, j, o, t and y). SI: signal intensity; FI: fluorescence intensity; PerCP. peridinin chlorophyll protein; FITC: fluorescein isothiocyanate; PE: phycoerythrin.

smoking history showed evidence of COPD. Subgroup analysis showed that the observed differences in DC characteristics between controls and patients were not influenced by the smoking history of the patients (data not shown). Of the 10 patients with sarcoidosis, six $(60 \%)$ had stage I disease and four $(40 \%)$ had stage II. Transbronchial biopsy specimens revealed noncaseating epitheloid cell granulomas in seven $(70 \%)$ of the patients. No differences were observed between stage I and stage II regarding differential cell counts or DC characteristics in patients with sarcoidosis (data not shown). In all patients with IPF, transbronchial biopsy specimens did not yield specific findings. In one patient, an open lung biopsy was performed, which confirmed the diagnosis of usual interstitial pneumonitis.

Of the 17 patients with pneumonia, 10 fulfilled the present criteria for immunosuppression. All of the patients with immunosuppression had received prednisolone directly before 
TABLE 2 Characteristics of participants and bronchoalveolar lavage fluid (BALF)

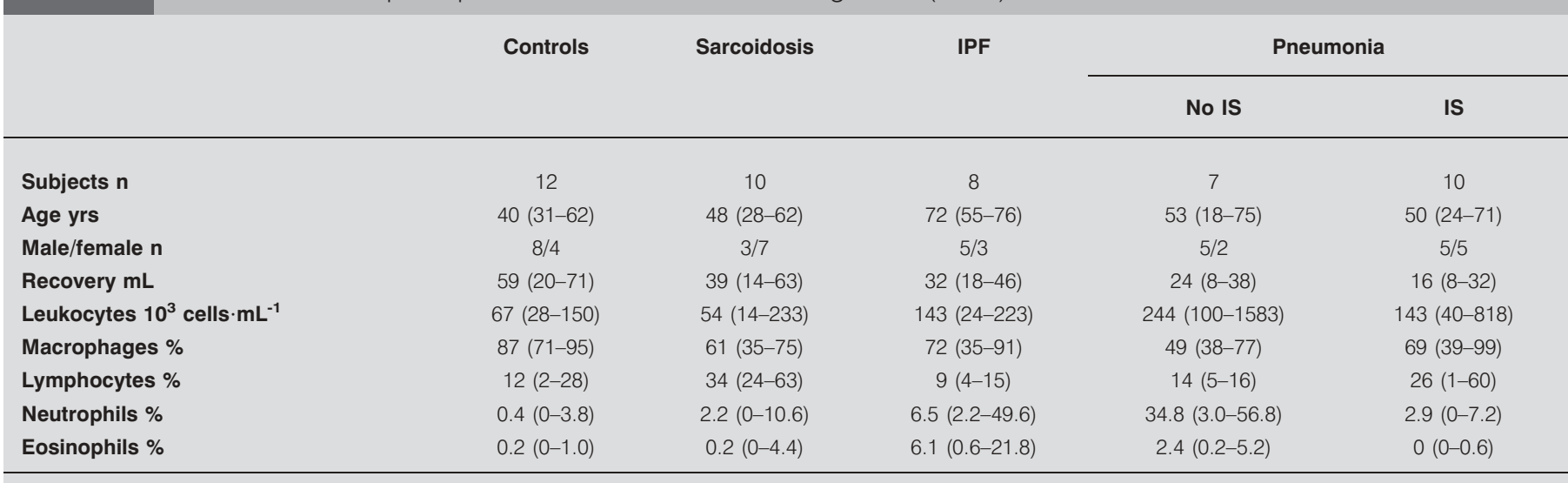

Data are presented as median (range), unless otherwise stated. Leukocyte subpopulations in BALF are shown as the percentage of all leukocytes. IPF: idiopathic pulmonary fibrosis; IS: immunosuppression.

or during the onset of symptoms. Two patients had received immunosuppressive therapy for renal transplants (triple therapy with prednisolone, cyclosporin and mycophenolate mofetil in both cases), and the other eight patients were treated for the following haematological disorders: multiple myeloma $(n=2)$, acute or chronic myeloid leukaemia $(n=3)$, acute or chronic lymphoblastic leukaemia $(n=2)$, and B-cell nonHodgkin's lymphoma $(n=1)$. Four of the haematological patients suffered from neutropenia following aggressive chemotherapy (which included prednisolone), and the other four were treated with prednisolone and cyclosporin after allogeneic stem cell transplantation and did not suffer from neutropenia. In all immunocompetent patients with pneumonia $(n=7)$, there was circumstantial evidence for a bacterial origin of the infection; bronchial secretions were purulent, and the patients responded promptly to antibiotic treatment. Microbiological evaluation of the BALF from this group yielded positive results in three out of seven cases (including Mycoplasma pneumoniae, Chlamydia pneumoniae and Legionella pneumophila). Patients with immunosuppression displayed bronchopneumonia or atypical infiltrates. Microbiological evaluation of the BALF from this group yielded positive results in seven out of 10 cases (including Pseudomonas aeruginosa, Aspergillus fumigatus, Candida albicans and Pneumocystis jiroveci).

\section{Dendritic cell subsets in BALF}

CD1a-positive DCs coexpressed CD11c in all BALF studied (data not shown). Total $\mathrm{pDC}$ and $\mathrm{mDC}$ percentages did not differ between patients with sarcoidosis and healthy controls. However, there was a significant increase in the number of CD1a-negative mDCs in patients with sarcoidosis compared to controls (table 3; fig. 2). DC subsets in BALF did not differ significantly between patients with IPF and controls (table 3; fig. 2). Immunocompetent patients with pneumonia were characterised by a unique increase in the number of BALF pDCs, as reflected by a strongly enhanced $\mathrm{pDC}: \mathrm{mDC}$ ratio. In contrast, immunocompromised patients with pneumonia displayed a decrease in the number of BALF pDCs compared to controls (table 3; fig. 2). The decrease in BALF mDC numbers in immunosuppressed patients was attributable to a decrease in the CD1a-positive subset (fig. 2).

\section{DC surface markers}

Surface marker expression on the BALF DCs of controls are shown in table 4 . The markers blood DC antigen (BDCA)-3 and -4

TABLE 3 Dendritic cell (DC) subsets in bronchoalveolar lavage fluid (BALF)

\begin{tabular}{|c|c|c|c|c|c|}
\hline & Controls & Sarcoidosis & IPF & No IS & IS \\
\hline pDCs \% BALF cells & $0.07(0.05-0.42)$ & $0.08(0.02-0.37)$ & $0.09(0.02-0.14)$ & $0.21(0.07-1.07)$ & $0.01(0.00-0.07)$ \\
\hline pDCs cells $\cdot \mathrm{mL} \mathrm{BALF}^{-1}$ & $53(20-170)$ & $73(10-200)$ & $115(10-250)$ & $1299(170-3000)$ & $3(0-410)$ \\
\hline mDCs \% BALF cells & $0.50(0.17-1.21)$ & $0.66(0.23-0.94)$ & $0.52(0.16-1.37)$ & $0.23(0.11-0.57)$ & $0.05(0.01-0.45)$ \\
\hline CD1a:mDC ratio" & $0.58(0.33-0.76)$ & $0.35(0.27-0.53)$ & $0.60(0.37-1.0)$ & $0.45(0.05-1.0)$ & $0.15(0.0-0.75)$ \\
\hline
\end{tabular}

Data are presented as median (range). IPF: idiopathic pulmonary fibrosis; IS: immunosuppression; pDC: plasmacytoid DC; mDC: myeloid DC. ${ }^{\#}:$ based on numbers of cells per $\mathrm{mL}$ of BALF. 

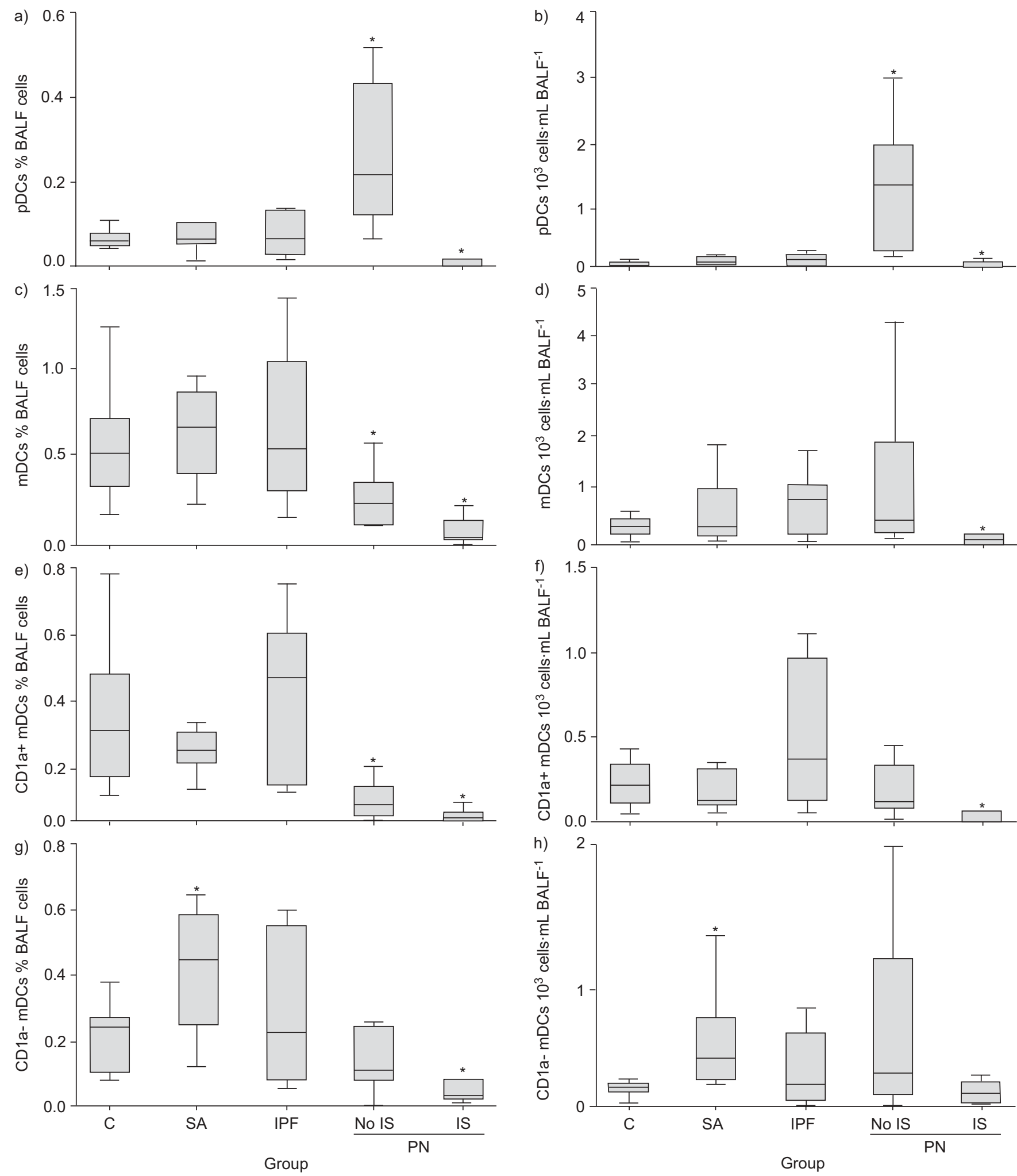

FIGURE 2. Dendritic cell (DC) subsets in bronchoalveolar lavage fluid (BALF) from various patient groups. Percentages $(a, c, e$ and $g$ ) and cell densities (b, $d$, $f$ and $h$ ) in BALF of: $a$ and b) plasmacytoid DCs (pDCs); $c$ and d) total myeloid DCs (mDCs); e and f) CD1a-positive mDCs; and g and h) CD1a-negative mDCs. The boxes show the median and interquartile ranges, and the vertical bars show the ranges of all values $<1.5$ interquartile ranges from the upper or lower quartile. C: control; SA: sarcoidosis; IPF: idiopathic pulmonary fibrosis; PN: pneumonia; IS: immunosuppression. *: $p<0.05$ versus controls. 


\begin{tabular}{|c|c|c|}
\hline \multirow[t]{2}{*}{ TABLE 4} & \multicolumn{2}{|c|}{$\begin{array}{l}\text { Surface molecules on dendritic cell (DC) in } \\
\text { bronchoalveolar lavage fluid from healthy } \\
\text { controls }\end{array}$} \\
\hline & mDCs & pDCs \\
\hline BDCA-1 & $60.7(46.5-74.8)$ & 0.0 \\
\hline BDCA-2 & 0.0 & $80.1(54.2-93.9)$ \\
\hline BDCA-3 & $70.1(62.6-86.1)$ & $95.1(83.1-100)$ \\
\hline BDCA-4 & $14.4(4.3-41.5)$ & $90.4(78.4-97.8)$ \\
\hline CD40 & $96.3(94.6-98.4)$ & $53.7(35.5-62.5)$ \\
\hline CD80 & $58.0(26.2-66.4)$ & NA \\
\hline CD83 & $23.4(17.7-39.2)$ & NA \\
\hline CD86 & $73.0(51.1-84.5)$ & NA \\
\hline \multicolumn{3}{|c|}{$\begin{array}{l}\text { Data are presented as median (range) percentage of marker-positive myeloid } \\
\text { DCs (mDCs) and plasmacytoid DCs (pDCs). The results of } 10 \text { subjects are } \\
\text { presented (the surface molecules were not analysed in two control subjects). } \\
\text { Numbers of pDCs were too low to reliably quantify the expression of the weakly } \\
\text { expressed molecules CD80, CD83 and CD86. BDCA: blood DC antigen; NA: } \\
\text { not analysable. }\end{array}$} \\
\hline
\end{tabular}

were not specific for mDCs or pDCs in BALF, neither in controls (table 4) nor in any of the patient groups (data not shown). CD80, CD86 and CD83 were weakly expressed on the pDCs of controls (table 4) and patients (data not shown). They could not be reliably quantified owing to the low number of pDCs in most patients and controls. In patients, the surface marker analysis included CD40, CD80, CD83 and CD86 on pDCs and mDCs, and BDCA- 1 and -3 on mDCs. However, surface molecules could not be reliably analysed in the BALF of the immunosuppressed group because of the very low numbers of $\mathrm{pDCs}$ and mDCs in most patients. Therefore, these data were omitted in the analyses. The expression of CD40 (on pDCs and mDCs), and of BDCA-3 on mDCs, did not differ between the analysable groups (data not shown). Patients with sarcoidosis displayed increased expression of CD80 on mDCs. In contrast, CD83 and CD86 levels were significantly decreased on mDCs (fig. 3). IPF was characterised by decreased expression of CD83 but normal expression of CD80, CD86 and BDCA1 on mDCs (fig. 3). Pneumonia without immunosuppression was associated with remarkably normal expression of DC surface molecules (fig. 3).

\section{Chemokines in BALF}

In order to elucidate the mechanisms of DC recruitment, concentrations of the DC-attracting chemokines CCL20 and CXCL10 were measured in the BALF supernatants of controls and patients (fig. 4). Both chemokines were undetectable in BALF supernatants from controls. CCL20 levels were increased in patients with IPF and in those with pneumonia (with or without immunosuppression). There was no significant difference in CCL20 level between patients with IPF and patients with pneumonia (fig. 4). In contrast, the chemokine CXCL10 was specifically and strongly elevated in patients with pneumonia. It is of note that this increase in CXCL10 concentration was not significantly influenced by immunosuppression (fig. 4). However, only in immunocompetent patients with pneumonia, but not in those with immunosuppression, was there a trend towards a positive correlation between CXCL10 concentration and pDC percentage in BALF $(r=0.63$; $\mathrm{p}=0.12)$.

\section{DISCUSSION}

The present study is the first to describe the distribution and surface marker expression of airway DCs in patients with sarcoidosis, IPF and pneumonia, and to compare these findings with those in healthy controls. Using a comprehensive flow cytometric method for the analysis of DC subsets in human BALF [3], a differential airway DC phenotype was identified in these diseases. In addition, differential expression of DC-attracting chemokines was found in the airways. Thus, the present study provides new insights into the characteristics and recruitment of airway DCs in inflammatory diseases of the human lung. Information regarding additional surface molecules expressed by airway DCs, which are important to the further characterisation of their function, could not be obtained in the present study due to the low DC counts in most BALF samples. Subsequent studies are, therefore, needed in order to reveal the functional properties of these DCs.

Current knowledge concerning the pathophysiology of pulmonary DCs is largely derived from animal studies [2]. Although there have been recent advances in the characterisation of DCs in human lung homogenates [16, 17] and lung parenchyma [18-20], the specific features of human airway DCs are still poorly understood. The currently available studies on airway DCs in human lung diseases were performed in patients with asthma, bronchiolitis and COPD $[3,8,10,11]$. In the present study, the occurrence and the phenotype of airway DCs were analysed in patients with sarcoidosis, IPF and pneumonia, and these findings compared with those in healthy volunteers. Some $(25 \%)$ of the patients, but none of the controls, had a positive smoking history, which might have affected the comparability between the groups. However, subgroup analyses suggested that the observed differences in DC phenotypes were not attributable to smoking.

In patients with asthma, it has recently been demonstrated that all CD1a-positive DCs in BALF coexpress CD11c, and it was concluded that CD1a-positive DCs represent a subset of human mDCs [3]. In addition, it was shown that two BDCAs (BDCA-3 and -4), which are specific for mDCs (BDCA-3) and pDCs (BDCA-4) in human blood [21], are not specific for the respective subsets in human BALF [3]. In the controls and patients of the present study, the coexpression of CD11c on all CD1a-positive DCs and the nonspecific expression of BDCA-3 and -4 were similar to the previous data [3]. Thus, the present observations in healthy volunteers and patients with sarcoidosis, IPF and pneumonia confirm three general findings from the previous study with asthma patients: 1) CD1a-positive DCs represent a subset of airway mDCs; 2) BDCA-3 is also expressed by airway $\mathrm{pDC}$ and thus not a specific marker for airway mDCs; and 3) BDCA-4 is also expressed by airway $\mathrm{mDCs}$ and thus not a specific marker for airway pDCs.

Accordingly, mDCs in human airways can be divided into CD1a-positive and -negative mDCs. This classification is substantiated by evidence suggesting a markedly different cytokine profile and T-cell stimulatory capacity of CD1a-positive 

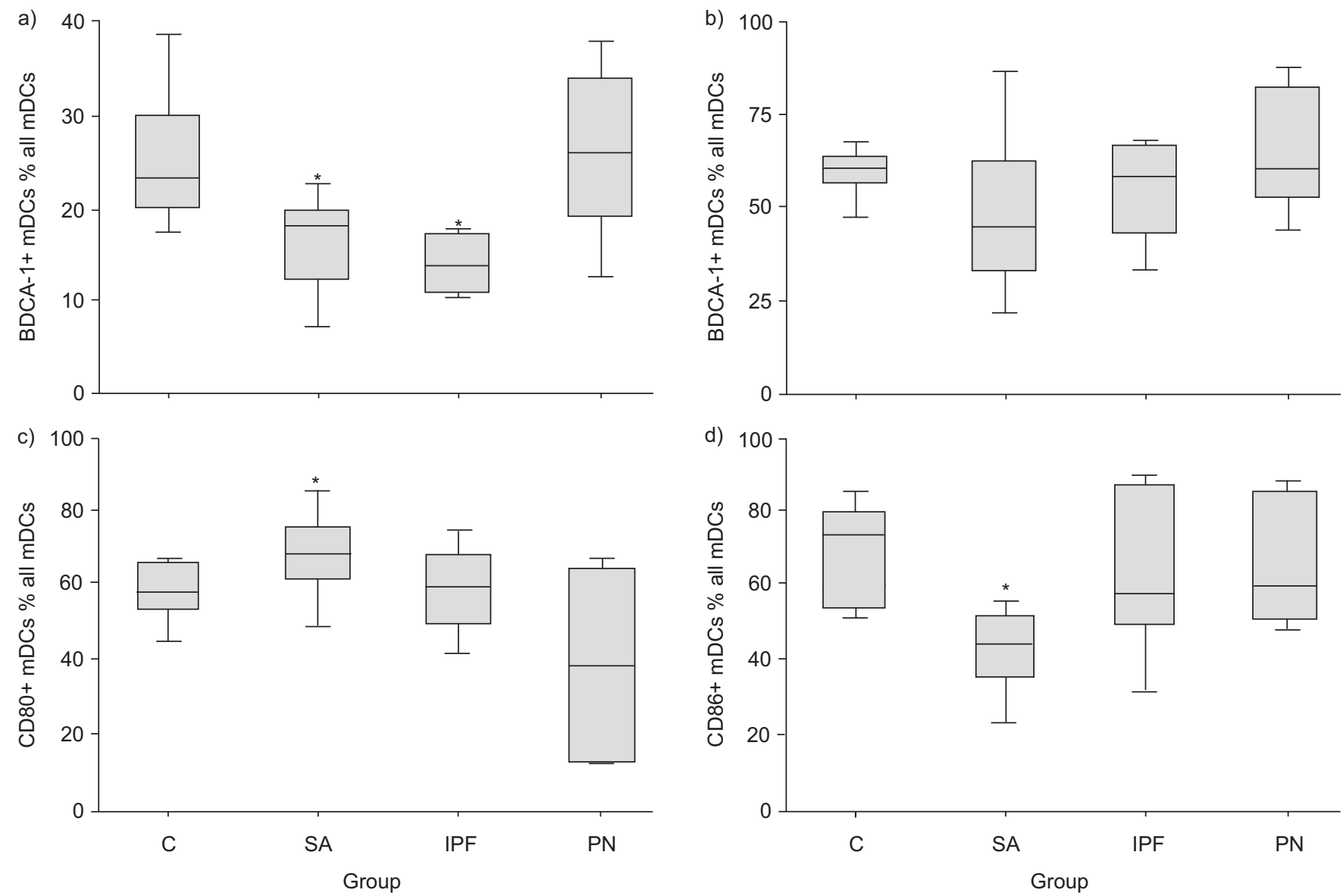

FIGURE 3. Surface molecule expression on myeloid dendritic cells (mDCs) in bronchoalveolar lavage fluid from various patient groups: a) CD83; b) blood dendritic cell antigen (BDCA) 1; c) CD80; and d) CD86. The boxes show the median and interquartile ranges, and the vertical bars show the ranges of all values $<1.5$ interquartile ranges from the upper or lower quartile. C: control; SA: sarcoidosis; IPF: idiopathic pulmonary fibrosis; PN: pneumonia. *: $p<0.05$ versus controls.

and -negative monocyte-derived DCs [5]. The 1996 study of VAN HAARST et al. [4] postulated that CD1a-positive and CD1a-negative accessory cells represent functionally different entities in human BALF, although the described population of CD1a-negative cells probably contained both DCs and monocytes. Therefore, functional analyses of purified CD1apositive and -negative DCs from human BALF are still needed in order to firmly establish the roles of these DCs in human airways. Nevertheless, the study of VAN HAARST et al. [4] showed that CD1a-negative accessory cells from human BALF are potent producers of the cytokines interleukin-1 and -6 and tumour necrosis factor (TNF)- $\alpha$, whereas CD1a-positive cells are not. In patients with sarcoidosis, a specific increase in CD1a-negative $\mathrm{mDC}$ numbers within the airways was observed. Interestingly, sarcoidosis is associated with enhanced production of interleukin- 1 and -6 and TNF- $\alpha$ [22, 23]. Thus, the observed increase in CD1a-negative mDCs might contribute to this cytokine profile. The hypothesis that CD1a-negative mDCs are involved in the pathogenesis of sarcoidosis is supported by immunohistochemical data showing that all DCs in human epithelioid cell granulomas (in both tumour-related sarcoid reactions and granulomas of patients with sarcoidosis) are CD1a-negative [24]. It is of note that the alveolar macrophages of patients with sarcoidosis can express costimulatory molecules (such as CD86 and CD80), which are almost absent on the macrophages of healthy controls. Given the expression of HLA-DR on alveolar macrophages, these data suggest that alveolar macrophages can acquire antigen-presenting capacities in patients with sarcoidosis [25]. Therefore, the relative contributions of DCs and macrophages to the pathogenesis of sarcoidosis remain to be elucidated [26]. Notably, it was observed that airway DCs in sarcoidosis express more CD80 than CD86, whereas macrophages in sarcoidosis were previously reported to express more CD86 than CD80 [25]. These findings point to different antigen-presenting properties of airway DCs and alveolar macrophages in sarcoidosis.

Evidence from animal models suggests that mDCs are potent inducers of pulmonary immune responses [27], whereas pDCs inhibit these responses [28]. The resulting concept that mDCs and $\mathrm{pDC}$ are antagonistic players, with pro-inflammatory $\mathrm{mDC}$ properties and tolerogenic PDC properties [29], has not yet been substantiated in humans. Recent reports demonstrate that acute upper respiratory tract infections [30] and acute allergic airway inflammation $[3,31]$ are associated with a strong influx of pDCs into human airways. This is in keeping with the present observation that immunocompetent patients with pneumonia displayed a marked increase in airway pDC numbers. In contrast, inflammatory disorders with a more chronic course, 

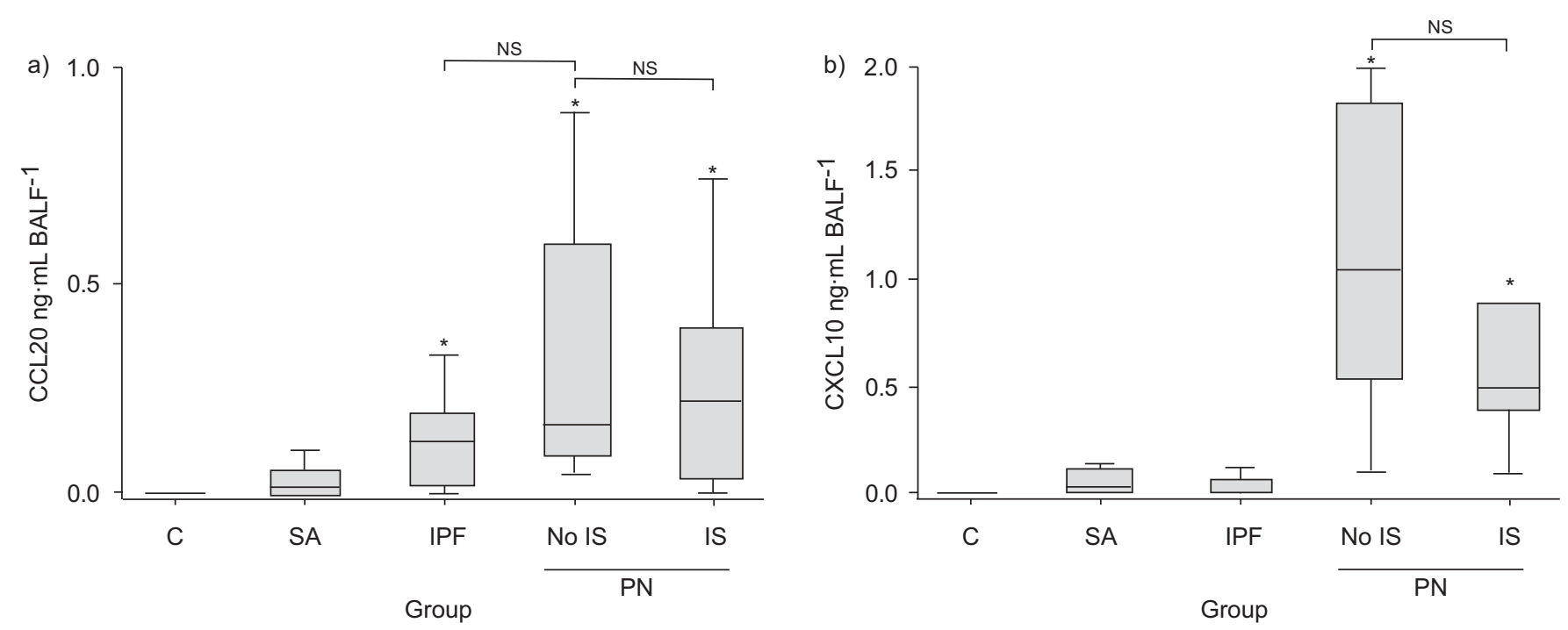

FIGURE 4. Chemokine concentrations in bronchoalveolar lavage fluid (BALF) from various patient groups: a) CC chemokine ligand (CCL)20; and b) CXC chemokine ligand (CXCL)10. The boxes show the median and interquartile ranges, and the vertical bars show the ranges of all values $<1.5$ interquartile ranges from the upper or lower quartile. C: control; SA: sarcoidosis; IPF: idiopathic pulmonary fibrosis; IS: immunosuppression; PN: pneumonia; NS: nonsignificant. *: $p<0.05$ versus controls.

such as sarcoidosis and IPF, were characterised by normal pDC numbers in the airways. Thus, human pDCs appear to be particularly attracted to sites of acute inflammation.

There are several potential stimuli that might account for this phenomenon. On the one hand, human pDCs can be recruited and activated by viral or bacterial antigens [32]. In the present study, immunocompetent patients with pneumonia showed evidence of a bacterial origin of the disease. In addition, a concomitant viral infection cannot be excluded in these patients. Thus, the strong increase in pDC numbers might be due to a release of bacterial and viral antigens in the airways. On the other hand, the strong influx of pDCs into the airways could also be due to endobronchial release of pDC-attracting chemokines. Indeed, strong and specific elevation of CXCL10 levels was found in the airways of patients with pneumonia. CXCL10, a ligand of the chemokine receptor CXCR3, which is strongly expressed on human pDCs, has been described as a crucial chemotactic factor for pDCs [33, 34]. Thus, increased endobronchial CXCL10 concentrations could recruit circulating pDCs into the airways. It is of note that several studies have demonstrated a strong influx of pDCs into the airways in the absence of infection [3,31]. It is therefore conceivable that the secretion of chemokines such as CXCL10, rather than the release of bacterial or viral antigens, is primarily responsible for $\mathrm{pDC}$ recruitment into the airways.

Another noteworthy result of the present study is the strong decrease in number of airway pDCs and CD1a-positive mDCs in immunocompromised patients with pneumonia. It is of note that these patients displayed endobronchial concentrations of CCL20, a potent chemotactic factor for mDCs [11], and CXCL10, a potent chemotactic factor for pDCs [33,34], similar to those observed in immunocompetent patients with pneumonia. These data suggest that immunocompromised patients with pneumonia do not lack the stimuli that attract DCs into the airways. Thus, it is more likely that immunosuppression impairs the survival of the DCs or the ability of the DCs to respond to chemotactic stimuli. It is of note that there was great heterogeneity in the underlying diseases in the immunocompromised patients, but treatment with prednisolone in $100 \%$ of cases. Thus, the effects observed in the immunosuppressed group appear to be related to the treatment, rather than the underlying disease. This is in line with reports demonstrating that immunosuppressive agents such as corticosteroids impact on the number and maturation of airway DCs [8, 35]. The finding that $\mathrm{pDCs}$ were more affected than mDCs might be due to the fact that pDCs are more susceptible to immunosuppressive therapies than mDCs [36]. Taken together, the present data suggest that immunocompromised patients with pneumonia are characterised by very low pDC counts in the airways, and that this phenomenon is not due to a decrease in pneumonia-associated chemotactic stimuli. Given the crucial role of $\mathrm{pDCs}$ in innate and adaptive defence mechanisms in the lung [32], it might be speculated that a reduced capacity to accumulate pDCs in the airways contributes to the increased susceptibility to respiratory tract infections in immunosuppressed patients.

In conclusion, the present study provides evidence that inflammatory diseases of the human lung are associated with a differential phenotype and recruitment of airway dendritic cells. Studies are warranted in order to define the role of these dendritic cells in the pathogenesis of human lung diseases.

\section{ACKNOWLEDGEMENTS}

The authors thank P. Thamm, J. Brandt, B. Voigt and G. Fastnacht for excellent technical assistance.

\section{REFERENCES}

1 Banchereau J, Briere F, Caux C, et al. Immunobiology of dendritic cells. Annu Rev Immunol 2000; 18: 767-811.

2 Vermaelen K, Pauwels R. Pulmonary dendritic cells. Am J Respir Crit Care Med 2005; 172: 530-551. 
3 Bratke K, Lommatzsch M, Julius P, et al. Dendritic cell subsets in human bronchoalveolar lavage fluid after segmental allergen challenge. Thorax 2007; 62: 168-175.

4 van Haarst JM, Verhoeven GT, de Wit HJ, et al. CD1a+ and CD1a- accessory cells from human bronchoalveolar lavage differ in allostimulatory potential and cytokine production. Am J Respir Cell Mol Biol 1996; 15: 752-759.

5 Chang CC, Wright A, Punnonen J. Monocyte-derived $\mathrm{CD}^{+} \mathrm{a}^{+}$and $\mathrm{CD}_{1} \mathrm{a}^{-}$dendritic cell subsets differ in their cytokine production profiles, susceptibilities to transfection, and capacities to direct Th cell differentiation. J Immunol 2000; 165: 3584-3591.

6 Lambrecht BN, Hammad H. Taking our breath away: dendritic cells in the pathogenesis of asthma. Nat Rev Immunol 2003; 3: 994-1003.

7 Bellini A, Vittori E, Marini M, et al. Intraepithelial dendritic cells and selective activation of Th2-like lymphocytes in patients with atopic asthma. Chest 1993; 103: 997-1005.

8 Moller GM, Overbeek SE, Van Helden-Meeuwsen CG, et al. Increased numbers of dendritic cells in the bronchial mucosa of atopic asthmatic patients: downregulation by inhaled corticosteroids. Clin Exp Allergy 1996; 26: 517-524.

9 Jahnsen FL, Moloney ED, Hogan T, et al. Rapid dendritic cell recruitment to the bronchial mucosa of patients with atopic asthma in response to local allergen challenge. Thorax 2001; 56: 823-826.

10 Todate A, Chida K, Suda T, et al. Increased numbers of dendritic cells in the bronchiolar tissues of diffuse panbronchiolitis. Am J Respir Crit Care Med 2000; 162: 148-153.

11 Demedts IK, Bracke KR, Van Pottelberge G, et al. Accumulation of dendritic cells and increased CCL20 levels in the airways of patients with chronic obstructive pulmonary disease. Am J Respir Crit Care Med 2007; 175: 998-1005.

12 Ota M, Amakawa R, Uehira K, et al. Involvement of dendritic cells in sarcoidosis. Thorax 2004; 59: 408-413.

13 Yoshinouchi T, Ohtsuki Y, Ueda R, et al. Myofibroblasts and S-100 protein positive cells in idiopathic pulmonary fibrosis and rheumatoid arthritis-associated interstitial pneumonia. Eur Respir J 1999; 14: 579-584.

14 Marchal-Somme J, Uzunhan Y, Marchand-Adam S, et al. Cutting edge: nonproliferating mature immune cells form a novel type of organized lymphoid structure in idiopathic pulmonary fibrosis. J Immunol 2006; 176: 5735-5739.

15 King TE. Clinical advances in the diagnosis and therapy of the interstitial lung diseases. Am J Respir Crit Care Med 2005; 172: 268-279.

16 Demedts IK, Brusselle GG, Vermaelen KY, et al. Identification and characterization of human pulmonary dendritic cells. Am J Respir Cell Mol Biol 2005; 32: 177-184.

17 Demedts IK, Bracke KR, Maes T, et al. Different roles for human lung dendritic cell subsets in pulmonary immune defense mechanisms. Am J Respir Cell Mol Biol 2006; 35: 387-393.

18 Bergeron A, El-Hage F, Kambouchner $\mathrm{M}$, et al. Characterisation of dendritic cell subsets in lung cancer micro-environments. Eur Respir J 2006; 28: 1170-1177.

19 Perros F, Dorfmuller P, Souza R, et al. Dendritic cell recruitment in lesions of human and experimental pulmonary hypertension. Eur Respir J 2007; 29: 462-468.
20 Lambrecht BN, van den Toorn LM. The pressure mounts on lung dendritic cells. Eur Respir J 2007; 29: 435-437.

21 Dzionek A, Fuchs A, Schmidt P, et al. BDCA-2, BDCA-3, and BDCA-4: three markers for distinct subsets of dendritic cells in human peripheral blood. I Immunol 2000; 165: 6037-6046.

22 Homolka J, Muller-Quernheim J. Increased interleukin 6 production by bronchoalveolar lavage cells in patients with active sarcoidosis. Lung 1993; 171: 173-183.

23 Prior C, Knight RA, Herold M, et al. Pulmonary sarcoidosis: patterns of cytokine release in vitro. Eur Respir J 1996; 9: 47-53.

24 Kurata A, Terado Y, Schulz A, et al. Inflammatory cells in the formation of tumor-related sarcoid reactions. Hum Pathol 2005; 36: 546-554.

25 Nicod LP, Isler P. Alveolar macrophages in sarcoidosis coexpress high levels of CD86 (B7.2), CD40, and CD30L. Am J Respir Cell Mol Biol 1997; 17: 91-96.

26 Nicod LP, Cochand L, Dreher D. Antigen presentation in the lung: dendritic cells and macrophages. Sarcoidosis Vasc Diffuse Lung Dis 2000; 17: 246-255.

27 Lambrecht BN, De Veerman M, Coyle AJ, et al. Myeloid dendritic cells induce Th2 responses to inhaled antigen, leading to eosinophilic airway inflammation. J Clin Invest 2000; 106: 551-559.

28 de Heer HJ, Hammad H, Soullie T, et al. Essential role of lung plasmacytoid dendritic cells in preventing asthmatic reactions to harmless inhaled antigen. J Exp Med 2004; 200: 89-98.

29 Hammad H, Lambrecht BN. Recent progress in the biology of airway dendritic cells and implications for understanding the regulation of asthmatic inflammation. J Allergy Clin Immunol 2006; 118: 331-336.

30 Hartmann E, Graefe H, Hopert A, et al. Analysis of plasmacytoid and myeloid dendritic cells in nasal epithelium. Clin Vaccine Immunol 2006; 13: 1278-1286.

31 Jahnsen FL, Lund-Johansen F, Dunne JF, et al. Experimentally induced recruitment of plasmacytoid (CD123high) dendritic cells in human nasal allergy. J Immunol 2000; 165: 4062-4068.

32 McKenna K, Beignon AS, Bhardwaj N. Plasmacytoid dendritic cells: linking innate and adaptive immunity. J Virol 2005; 79: 17-27.

33 Kohrgruber N, Groger M, Meraner P, et al. Plasmacytoid dendritic cell recruitment by immobilized CXCR3 ligands. J Immunol 2004; 173: 6592-6602.

34 Cravens PD, Hayashida K, Davis LS, et al. Human peripheral blood dendritic cells and monocyte subsets display similar chemokine receptor expression profiles with differential migratory responses. Scand I Immunol 2007; 65: 514-524.

35 Verhoeven GT, Van Haarst JM, De Wit HJ, et al. Glucocorticoids hamper the ex vivo maturation of lung dendritic cells from their low autofluorescent precursors in the human bronchoalveolar lavage: decreases in allostimulatory capacity and expression of CD80 and CD86. Clin Exp Immunol 2000; 122: 232-240.

36 Hackstein H, Renner FC, Bohnert A, et al. Dendritic cell deficiency in the blood of kidney transplant patients on long-term immunosuppression: results of a prospective matched-cohort study. Am J Transplant 2005; 5: 2945-2953. 